\title{
Salarios mínimos: desigualdad y desarrollo ${ }^{1}$
}

Minimum Wages: Inequality and Development

\section{Saúl Escobar Toledo*}

* Profesor-Investigador, Instituto Nacional de

Antropología e Historia

«saulescoba@gmail.com»

Journal of Economic Literature (JEL):

E24, D63, O2

Palabras clave:

Salarios

Desigualdad

Desarrollo

Keywords:

Wage

Inequality

Development

\section{Resumen}

Se analiza la evolución de los salarios mínimos en México durante las últimas décadas y se muestra su impacto en el crecimiento y en el empleo. Se señala que alrededor de $40 \%$ de la población mexicana está en esa condiciones de pobreza por razones laborales y el autor muestra cómo la caída del salario mínimo ha ido al parejo del conjunto de los salarios. Ello se refleja en la concentración del ingreso y en su distribución. Por lo tanto sostiene que los salarios mínimos han sido un indicador de cómo se han movido el conjunto de la estructura salarial: su aumento estimula una economía en crecimiento, mientras que su caída genera una economía en crisis. Mantener deprimido el salario mínimo ha ahondado las brechas salariales y la desigualdad. Por ello sugiere un cambio en la política salarial, pues podría inducir una nueva dinámica en la que un crecimiento de la demanda interna incremente la productividad y los niveles de empleo y de ingresos. Tal estrategia permitiría un mayor bienestar y una disminución efectiva de la pobreza y la desigualdad; y con ello se impactaría positivamente en el nivel de vida de los trabajadores.

\begin{abstract}
It analyzes the evolution of the minimum wages in Mexico during the last decades and shows its impact on growth and employment. It is noted that around $40 \%$ of the Mexican population is in such conditions of poverty for employment reasons, the author shows how the fall of the minimum wage has been parallel with the set of wages. This is reflected in the concentration of income and its distribution. Therefore argues that the minimum wage had been an indicator of how they moved the whole of the wage structure: its increase stimulates a growing economy, while its fall generates an economy in crisis. Maintain depressed the minimum wage has deepened the gaps in wages and inequality. He therefore suggested a change in the wage policy, because it might induce a new dynamic in which a growth of domestic demand increase productivity and levels of employment and income. Such a strategy would allow a greater well-being and a real reduction in poverty and inequality; and this could dampen positively in the standard of living of the workers.
\end{abstract}

\footnotetext{
${ }^{1}$ Dirección de Estudios Históricos, Instituto Nacional de Antropología e Historia
} 
a evolución de los salarios mínimos en las últimas décadas puede observarse claramente a partir de los datos oficiales. De acuerdo a la serie estadística más larga disponible para el periodo más reciente, publicados por el Banco de México e INEGI, se pueden distinguir los siguientes periodos:

De 1969 a 1977, el salario mínimo real crece a 5.7\% anual llegando a su punto más alto en este último año. Luego seguirá un periodo más corto, de 1977 a 1982 en que el salario mínimo tiene un ligero decrecimiento de 1.3\% anual. En cambio, entre 1982 y 1992 se da la peor caída del salario mínimo en la historia del país: 6.36\% anual acumulando un baja de 63.6 por ciento.

Posteriormente entre 1992 y 1995 la caída es un poco más suave, de $2 \%$ anual para luego volver a incrementarse entre 1995 y 2003 a un ritmo negativo de $2.5 \%$ anual. Finalmente entre 2003 y 2014 el salario mínimo se mantiene constante, ni crece ni decrece.

\begin{tabular}{|c|c|c|}
\hline \multicolumn{3}{|c|}{$\begin{array}{l}\text { Cuadro } 1 \\
\text { Salario mínimo real } 1969-2014\end{array}$} \\
\hline Periodo & Acumulado \% & Crecimiento/decrecimiento anual \\
\hline $1969-1977$ & 45.8 & 5.7 \\
\hline $1977-1982$ & -6.5 & -1.3 \\
\hline $1982-1992$ & -63.6 & -6.36 \\
\hline $1992-1995$ & -6.2 & -2.0 \\
\hline $1995-2003$ & -20.2 & -2.5 \\
\hline 2003- 2014 & 0 & 0 \\
\hline $1977-2014$ & -74.5 & -2.0 \\
\hline
\end{tabular}

Fuente: INEG//Banco de México

Garavito2 (2013) elaboró una gráfica sobre la caída del salario mínimo que resulta elocuente.

2 Rosa Albina Garavito, "Recuperar el salario real: un objetivo impostergable" Friedrich Ebert Stiftung (FES), México, 2013, p. 11. 


\section{Gráfica 1}

Salario mínimo real

Pesos de 2010

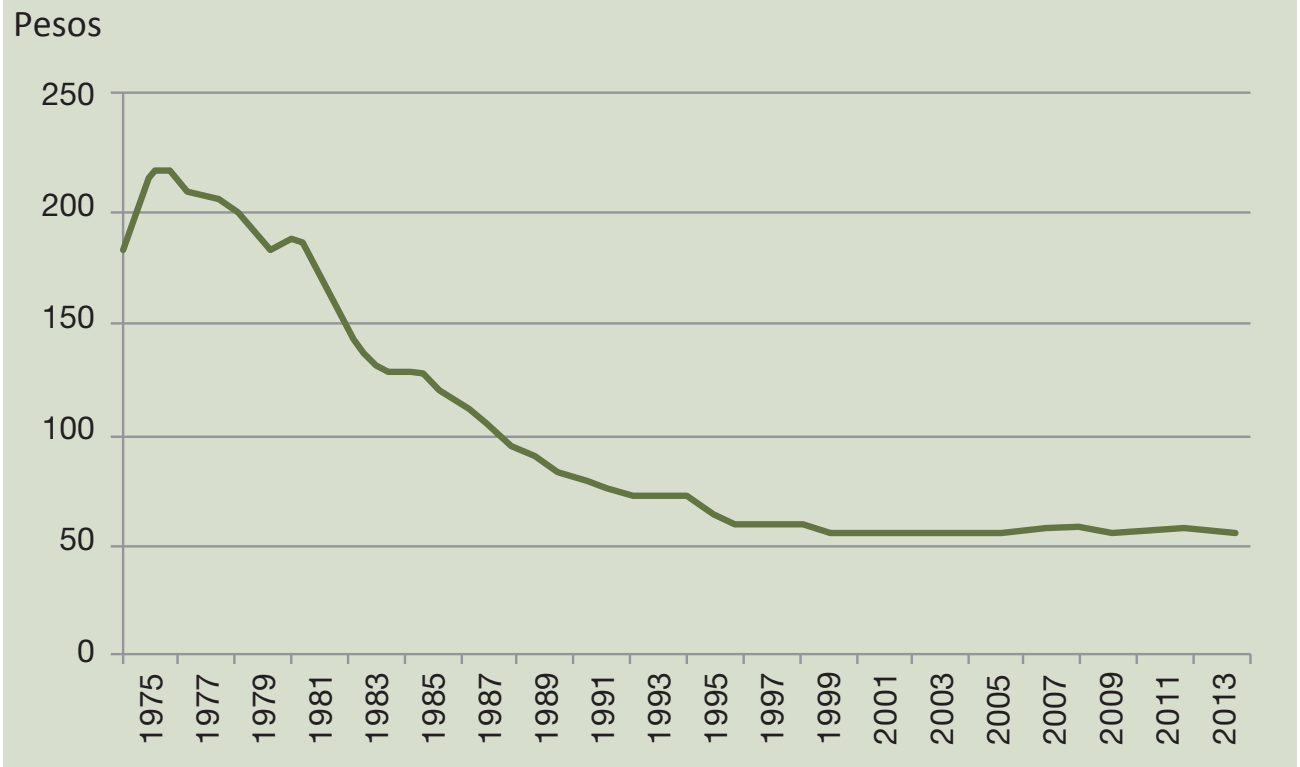

Fuente: Rosa Albina Garavito, elaboración propia con datos de CNSM e INEGI Nota: Corresponde al salario mínimo general promedio

La evolución de los salarios mínimos desde 1977 hasta el presente contrasta con su comportamiento en la etapa anterior, particularmente la que corre entre 1954 (año de la devaluación del peso) y 1977. Fue también un largo periodo de crecimiento lo que permitiría un aumento sostenido del empleo urbano.

Según Bortz, ${ }^{3}$ el salario mínimo legal en el Distrito Federal aumentó en términos reales de 1.09 pesos en 1955 a 3.15 en 1975 (p. 278, 1988). Lo que es más importante, las remuneraciones de los trabajadores como porcentaje del PIB también aumentan de 26.9 a $38.1 \%$ en ese mismo periodo.

\begin{tabular}{|c|c|c|}
$\begin{array}{l}\text { Cuadro } 2 \\
\text { Salario mínimo legal en el Distrito Federal y remuneraciones totales como \% del PIB }\end{array}$ \\
\hline & \multicolumn{2}{c|}{$\begin{array}{c}\text { Salario mínimo legal en el DF } \\
\text { Salario diario real (pesos de 1939) }\end{array}$} \\
\hline 1939 & 2.50 & $\begin{array}{c}\text { Remuneraciones totales como } \\
\text { porcentaje del PIB }\end{array}$ \\
\hline 1951 & 0.82 & 30.5 \\
\hline 1955 & 1.09 & 23.4 \\
\hline 1960 & 1.51 & 26.9 \\
\hline 1966 & 2.26 & 31.2 \\
\hline 1970 & 2.70 & 33.5 \\
\hline 1975 & 3.15 & 35.7 \\
\hline
\end{tabular}

Fuente: Jeff Bortz, Los salarios industriales en la Ciudad de México, 1988.

3 Jeffry Lawrence Bortz, Los salarios industriales en la Ciudad de México 1939-1975. FCE, 1988, p. 278 . 
Carlos Tello4 (p. 220, 2010) muestra un crecimiento del salario mínimo real en todo el país con tasas muy elevadas sobre todo entre 1959 y 1964 pues llegan a alcanzar casi $8 \%$ anual (cuadro 3). Este crecimiento se sostiene según el autor hasta 1981-82 en el caso de los salarios contractuales: (cuadro 4).

\section{Cuadro 3}

Salario Mínimo real, tasa de crecimiento anual (\%)

\begin{tabular}{|l|l|}
\hline $1953-58$ & 4.22 \\
\hline $1959-64$ & 7.73 \\
\hline $1965-70$ & 4.70 \\
\hline
\end{tabular}

Fuente: Carlos Tello."Sobre la desigualdad..." Con base en información de INEGI, Comisión Nacional de los salarios Mínimos y Banco de México.

\section{Gráfica 2}

Salario mínimo real

(1939-1975)

Pesos de 1939

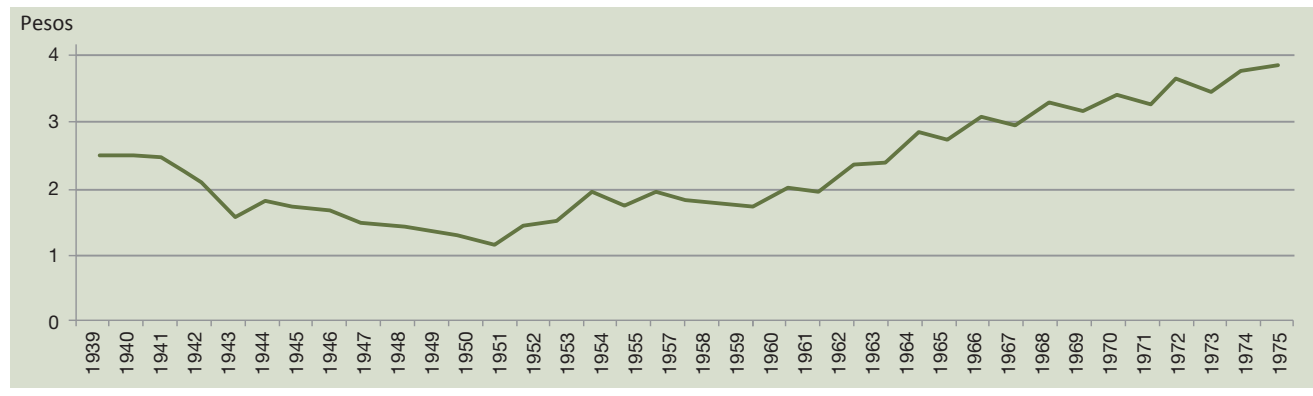

Fuente: elaboración propia con datos de CNSM, Banxico e INEGI. Garavito, "Recuperar el salario..."

\begin{tabular}{|c|c|c|}
\hline \multicolumn{3}{|l|}{$\begin{array}{l}\text { Cuadro } 4 \\
\text { Salarios mír }\end{array}$} \\
\hline & Mínimos & Medios contractuales \\
\hline 1970 & 44.57 & n.d. \\
\hline 1975 & 48.76 & 61.17 \\
\hline 1976 & 54.33 & 61.98 \\
\hline 1981 & 48.35 & 61.83 \\
\hline 1982 & 42.73 & 67.67 \\
\hline
\end{tabular}

Fuente: Carlos Tello, Sobre la desigualdad en México, 2010.

Otra gráfica elaborada por Rosa Albina Garavito refleja el crecimiento del salario mínimo real entre 1939 y 1975, coincidiendo con las tendencias anotadas por los autores citados más arriba.

Al parejo de un aumento de los salarios mínimos y contractuales se presentó, como ya vimos, una mejor distribución del ingreso entre los factores de la producción. A mayor abundamiento, Reyes Heroles 5 (1981, p. 2) señala que la participación de los asalariados en el ingreso total aumentó rápidamente en México entre 1950 y

4 Tello, Carlos Sobre la desigualdad en México, unAm, Facultado de Economía, México, 2010.

5 Jesús Reyes Heroles G.G., "La Distribución de los Ingresos por Trabajo en México”. Banco de México, 1981. 
1976, pasando de 27.3 a 49.4\% en 1976. David Márquez 6 (2007) calcula que la remuneración de los asalariados llegó hasta casi 44\% en 1976 (cuadro 5).

\begin{tabular}{|c|c|c|}
\hline \multicolumn{3}{|c|}{$\begin{array}{l}\text { Cuadro } 5 \\
\text { Remuneración de los asalariados y excedente de operación } \\
\text { Ingreso nacional disponible }=100\end{array}$} \\
\hline Año & Remuneración de asalariados & Excedente de operación \\
\hline 1970 & 38.1 & 57.8 \\
\hline 1976 & 43.5 & 52.9 \\
\hline 1982 & 41.6 & 54.6 \\
\hline \multicolumn{3}{|c|}{$\begin{array}{l}\text { Fuente: Sistema de cuentas nacional; no incluye: Ingresos netos factoriales del resto del mundo; } \\
\text { impuestos indirectos; subsidios y consumo de capital. Cf. David Márquez, "La distribución del } \\
\text { ingreso..." }\end{array}$} \\
\hline
\end{tabular}

El crecimiento económico y el aumento en los salarios reales y el empleo, sobre todo urbanos, parecía anunciar un país que se dirigía hacia una modernidad compartida. Pero la verdad era que este crecimiento se basaba, en mucho, en el sacrificio del campo. La agricultura mexicana aportó cuantiosos recursos para el desarrollo urbano e industrial. El otro lado de la moneda de la modernidad del milagro mexicano se reflejaba en los altos niveles de pobreza y en el atraso que sufrían particularmente las áreas rurales.

Además de esta tendencia, el otro ingrediente negativo de este periodo fue el autoritarismo. La oposición política era muy reducida y su influencia casi marginal en el Congreso de la Unión y en los gobiernos estatales y municipales. Los sindicatos se controlaban con base en un sistema corporativo que se distinguía por la ausencia de democracia. ${ }^{7}$ La movilización social, aunque presente todos estos años, no era tolerada.

\section{La situación actual: salario mínimo y pobreza}

Si la etapa que culminó en 1977 se distinguió por el crecimiento del salario mínimo legal y de la masa salarial total, la más reciente se ha caracterizado por su contrario: la caída del salario mínimo y, en casi toda esta etapa (hasta el año 2000), de los salarios en su conjunto, lo que se ha traducido en un aumento de la pobreza y la desigualdad en México ya no sólo en el campo sino en el conjunto del país.

Ello es explicable pues como ha señalado la CEPAL (2014, p. 140):

El mundo del trabajo cumple un rol fundamental en la generación y perpetuación de las desigualdades que caracterizan a las sociedades latinoamericanas. En este espacio se

6 David Márquez, "La Distribución del Ingreso y el gasto en México", La Jornada, 8 de octubre de 2007.

7 Para un recuento histórico del sindicalismo mexicano y una reflexión sobre la relación entre el sindicalismo corporativo y el Estado, cf. Saúl Escobar Toledo, Los Trabajadores en el Siglo xx. Sindicatos, Estado y Sociedad en México 1907-2004, unAm, 2006. 
generan la mayor parte de los ingresos de los hogares de la región y las desigualdades inherentes a su distribución...8

De acuerdo a los estudios de $\operatorname{conEVAL}^{9}$ (2014) los trabajadores que reciben un salario mínimo diario están por debajo de los mínimos de bienestar. Por lo menos entre 2005 y 2014 (años que hay información disponible) el salario mínimo no ha sido suficiente para cubrir los necesidades elementales en las zonas urbanas y su cobertura ha oscilado entre 81 y $77 \%$. Más grave todavía, si consideramos no sólo al trabajador individualmente sino a su familia, los índices se desploman. Según los criterios de la CEPAL para que un hogar de cuatro miembros, dos de ellos perceptores de ingresos, logre superar el umbral de la pobreza, la relación entre el salario mínimo y el valor de la línea de la pobreza debe ser superior a dos. ${ }^{10}$

Con base en lo anterior, puede señalarse que en México, sólo los trabajadores que reciben un salario mínimo en las áreas rurales cumplen este criterio y únicamente para adquirir la canasta alimentaria pero no para su bienestar general. Aún más, los trabajadores que perciben dos salarios mínimos en las áreas urbanas tampoco superan el nivel de la pobreza desde el punto de vista de su bienestar general pues cubriría tan sólo 80\% de sus necesidades en 2013 (cuadro 6 ). Así, más de 10 millones de trabajadores (5.3 en las áreas más urbanizadas y 4 en las menos urbanizadas y un millón en las áreas rurales) se encontraban en 2013 en una situación de pobreza. Ello quiere decir que alrededor de $40 \%$ de la población mexicana está en esa condición por razones laborales (salariales) (cuadro 7).

Cuadro 6

Índice de pobreza de uno y dos salarios mínimos: 2005 y 2013

\begin{tabular}{|c|c|c|c|c|}
\hline & \multicolumn{2}{|c|}{$\begin{array}{c}\text { Bienestar mínimo } \\
\text { (canasta alimentaria) }\end{array}$} & $\begin{array}{c}\text { Línea de bienestar (canasta alimen- } \\
\text { taria y no alimentaria) }\end{array}$ \\
\hline Año / SMD & rural & urbano & rural & urbano \\
\hline 2005 / 1 SMD & 2.75 & 1.9 & 1.3 & 0.81 \\
\hline $2005 / 2$ SMD & 5.50 & 3.8 & 2.7 & 1.60 \\
\hline $2013 / 1$ SMD & 2.30 & 1.6 & 1.2 & 0.79 \\
\hline $2013 / 2$ SMD & 4.60 & 3.3 & 2.5 & 1.60 \\
\hline
\end{tabular}

Nota: el índice mínimo para el sostenimiento del trabajador y su familia debe ser superior a 2 calculado con base en salarios en pesos corrientes, SMD promedio nacional.

Fuente: elaboración propia con datos de CONEVAL e INEGI.

Valores mensuales per cápita a precios corrientes, SMD promedio.

Fuente: elaboración propia con datos de CONEVAL e INEGI.

8 CEPAL, Pactos para la Igualdad. Hacia un futuro sostenible, Trigèsimo quinto período de sesiones de la CEPAL, mayo de 2014, p. 140.

9 Consejo Nacional de Evaluación de la Política Social (CONEVAL) "Índice de Tendencia laboral de la Pobreza, 2014".

10 CEPAL, "Pactos para la igualdad...", op. cit. p. 154. 


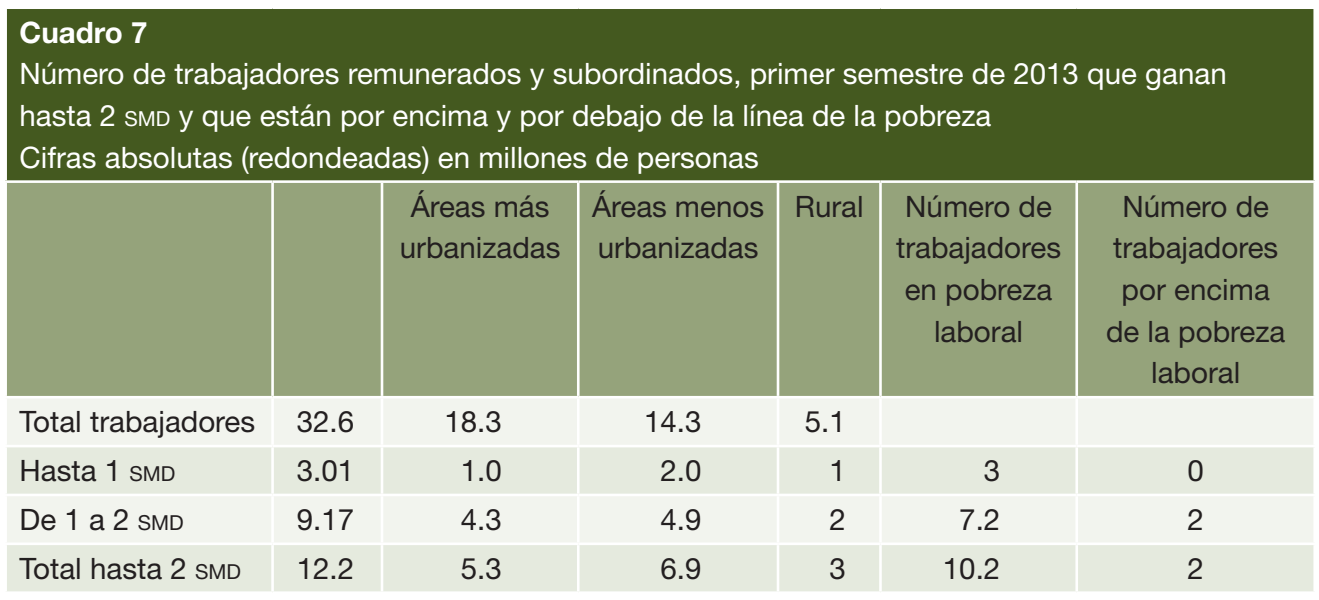

Nota: los trabajadores rurales forman parte del grupo de trabajadores de las áreas menos urbanizadas según la metodología del INEGI.

Fuente: elaboración propia con datos de CONEVAL e INEGI.

\section{Características de los trabajadores de salario mínimo}

Los trabajadores que ganan hasta un salario mínimo diario (SMD) han representado entre $11 \%$ (en 2005) y casi $9 \%$ (en 2014) ${ }^{11}$ del total (cuadro 8). Pero, observando con más detalle las estadísticas disponibles se puede afirmar que los trabajadores que con este nivel de ingresos se ubican principalmente en las áreas menos urbanizadas: más de 66\%. De ellos, casi la mitad son trabajadores rurales (cuadro 9). En la Ciudad de México por comparación el número de trabajadores que recibía un SMD representaban $7 \%$ y $6.4 \%$ del total en esos mismos años. De la misma manera, puede advertirse que los trabajadores que reciben un salario mínimo tienen distinta composición según el género: la proporción de mujeres que ganan un SMD es mayor respecto al total que en el caso de los hombres (ver cuadro 10).

\begin{tabular}{|c|c|c|c|}
\hline \multicolumn{4}{|c|}{$\begin{array}{l}\text { Cuadro } 8 \\
\text { Población ocupada y subordinada por ingresos al primer trimestre de cada año }\end{array}$} \\
\hline Año & $\begin{array}{l}\text { Total ocupada } \\
\text { subordinada }\end{array}$ & Hasta 1 SMD & $\%$ \\
\hline 2005 & 26933613 & 2998970 & 11.1 \\
\hline 2013 & 32650414 & 3014548 & 9.2 \\
\hline 2014 & 33486422 & 2948947 & 8.8 \\
\hline
\end{tabular}

Fuente: INEGI.

11 Para los fines de este trabajo, sólo lo hemos tomado en cuenta a los trabajados asalariados. Si se incluyera al total de la población ocupada la cifra de trabajadores que gana el salario mínimo sería mayor, alrededor de $13 \%$ en los últimos años. 
Cuadro 9

Trabajadores remunerados y subordinados, que ganan un SMD en áreas más urbanizadas, menos urbanizadas y rurales, primer semestre de 2013

Cifras absolutas en millones de personas

\begin{tabular}{|l|c|c|c|c|c|c|}
\hline & & $\begin{array}{c}\text { Áreas más } \\
\text { urbanizadas }\end{array}$ & $\begin{array}{c}\text { Áreas menos } \\
\text { urbanizadas }\end{array}$ & $\begin{array}{c}\text { Menos } \\
\text { urbanizadas/ } \\
\text { total \% }\end{array}$ & Rural & $\begin{array}{c}\text { Rural / menos } \\
\text { urbanizadas \% }\end{array}$ \\
\hline Total trabajadores & 32.6 & 18.3 & 14.3 & 43.9 & 5.07 & 35.5 \\
\hline Hasta 1 SMD & 3.01 & 1.0 & 2.01 & 66.8 & 0.97 & 48.2 \\
\hline
\end{tabular}

Nota: los trabajadores rurales forman parte del grupo de trabajadores de las áreas menos urbanizadas según la metodología del INEGI.

Fuente: INEGI.

\section{Cuadro 9}

Mujeres y hombres trabajadores remunerados y subordinados en $\%$ y millones de personas

\begin{tabular}{|l|c|c|}
\hline Mujeres & $\%$ & Absolutos \\
\hline Total & 100 & 12.18 \\
\hline Hasta 1 SMD & 12.4 & 1.51 \\
\hline De 1 a 2 SMD & 31.9 & 3.89 \\
\hline Total hasta 2 SMD & 44.3 & 5.40 \\
\hline Hombres & 100 & 20.47 \\
\hline Hasta 1 SMD & 7.3 & 1.5 \\
\hline De 1 a 2 SMD & 25.8 & 5.28 \\
\hline Total hasta 2 SMD & 33.1 & 6.78 \\
\hline
\end{tabular}

Fuente : INEGI

\section{Segmentación del mercado laboral}

El mercado laboral mexicano se encuentra muy segmentado. Podemos hablar de dos grupos de trabajadores que se distinguen por características tales como el acceso a las instituciones de salud, las prestaciones laborales, la disponibilidad de un contrato escrito y si éste es temporal o de base. Casi la mitad de los trabajadores mexicanos (asalariados) pertenecen al segmento más desfavorecido que se caracteriza por no tener acceso a las instituciones de salud, carecen de prestaciones (como aguinaldo, vacaciones, fondos de pensiones y de vivienda), no tienen un contrato escrito y si lo tienen son temporales. Pero es posible señalar, además, que otra característica muy importante que los distingue y es que estos trabajadores laboran en micronegocios o pequeños establecimientos (ver cuadro 10). 


\begin{tabular}{|c|c|c|}
\hline \multicolumn{3}{|l|}{$\begin{array}{l}\text { Cuadro } 10 \\
\text { Trabajadores subordinados y remunerados }\end{array}$} \\
\hline & 2005 & 2014 \\
\hline 4.4. Cond. de acceso a las instituciones de salud & 100.0 & 100.0 \\
\hline Con acceso & 54.3 & 54.0 \\
\hline Sin acceso & 44.8 & 45.3 \\
\hline No especificado & 0.9 & 0.7 \\
\hline $\begin{array}{l}\text { 4.5. Prestaciones laborales (sin considerar el acceso a las institucio- } \\
\text { nes de salud) }\end{array}$ & 100.0 & 100.0 \\
\hline Con prestaciones & 61.6 & 62.8 \\
\hline Sin prestaciones & 37.9 & 36.7 \\
\hline No especificado & 0.4 & 0.5 \\
\hline 4.6. Disponibilidad de contrato escrito & 100.0 & 100.0 \\
\hline Con contrato escrito & 50.3 & 53.2 \\
\hline Temporal & 18.0 & 17.3 \\
\hline De base, planta o por tiempo indefinido & 81.4 & 82.2 \\
\hline Contrato de tipo no especificado & 0.6 & 0.5 \\
\hline Sin contrato escrito & 48.8 & 46.0 \\
\hline No especificado & 0.9 & 0.8 \\
\hline 5.1. Tamaño de la unidad económica & 100.0 & 100.0 \\
\hline Micronegocios & 27.1 & 25.5 \\
\hline Sin establecimiento & 41.1 & 39.5 \\
\hline Con establecimiento & 58.9 & 60.5 \\
\hline Pequeños establecimientos & 23.9 & 23.4 \\
\hline Suma de micro y pequeños negocios & 51.0 & 48.9 \\
\hline Medianos establecimientos & 16.6 & 15.9 \\
\hline Grandes establecimientos & 15.2 & 15.1 \\
\hline Gobierno & 7.6 & 7.6 \\
\hline Otros & 7.9 & 7.9 \\
\hline No especificado & 1.7 & 4.6 \\
\hline
\end{tabular}

Fuente: elaboración propia con datos de INEGI.

Los trabajadores que reciben hasta dos SMD y una buena parte de los que ganan hasta tres SMD (los que se encuentran en las áreas menos urbanizadas y rurales) formarían parte de este segmento de trabajadores sin protección social y que labora en condiciones precarias 12 (ver cuadros 11 y 12 ).

12 Vale la pena subrayar que la estadísticas oficiales del INEGi tienen un alto nivel de imprecisión pues la categoría no especificado llegaba en 2014 a 11\% del total y casi a 15\% en las zonzas urbanas (cuadro 19). 


\section{Cuadro 11}

Trabajadores remunerados por nivel de ingresos 2005-2014\%

Trabajadores subordinados y remunerados por Primer trimestre 2005

Primer trimestre 2014

Nivel de ingresos

100.0

100.0

Hasta un salario mínimo

11.1

8.8

Más de 1 hasta 2 salarios mínimos

28.7

28.0

Suma $1+2$ SMD

39.8

36.8

Más de 2 hasta 3 salarios mínimos

24.3

27.4

Hasta 3 SMD

64.1

64.2

Más de 3 hasta 5 salarios mínimos

20.0

17.1

Más de 5 salarios mínimos

10.8

No especificado

5.1

11.0

Fuente: elaboración propia con datos de INEGI.

\section{Cuadro 12}

Trabajadores remunerados y subordinados que ganan hasta un salario mínimo y hasta dos sala-

rios mínimos según área más urbanizada, menos urbanizada y rural, y no especificado. En \% del

total de cada categoría. Primer trimestre de 2013

\begin{tabular}{|l|c|c|c|c|}
\hline & \% del total & Áreas más urbanizadas & Áreas menos urbanizadas & Rural \\
\hline Hasta 1 SMD & 9.2 & 5.4 & 14.1 & 19.1 \\
\hline 1-2 SMD & 28.1 & 23.4 & 34.1 & 39.6 \\
\hline Total hasta 2 SMD & 37.3 & 28.8 & 48.2 & 58.7 \\
\hline No especificado & 10.9 & 14.6 & 6.0 & 4.4 \\
\hline
\end{tabular}

Nota: los trabajadores rurales forman parte del grupo de trabajadores de las áreas menos urbanizadas, según la metodología del INEGI.

Fuente: INEGI, ENOE.

Por su parte, un estudio de McKinsey 13 considera que el empleo "informal" entendido aquí como el que otorgan las empresas que emplean trabajadores que no cumplen con la ley pues no les otorgan seguridad social, lo que en otros términos se conoce como empleo precario, está muy relacionado con la región, la edad, el nivel de educación y el sector productivo: aumenta en los estados más pobres como Oaxaca y Guerrero, en los grupos etarios más viejos y más jóvenes, en el sector primario y en los niveles de educación más bajos. (cuadro 13)

\section{Cuadro 13}

$\%$ de informalidad en el total de la fuerza de trabajo en rubros seleccionados

\begin{tabular}{|l|c|c|c|}
\hline Estados & Edad & Sector económico & Nivel de educación \\
\hline Oaxaca & +65 & Primario & Inferior a la primaria \\
\hline $81 \%$ & $80 \%$ & $90 \%$ & $87 \%$ \\
\hline Guerrero & $14-24$ & Servicios & Primaria \\
\hline $79 \%$ & $71 \%$ & $82 \%$ & $77 \%$ \\
\hline
\end{tabular}

Fuente: "A tale of two Mexicos..." Instituto McKinsey

13 Eduardo Bolio y Jaana Temes, et al., A tale of two Mexicos: Growth and prosperity in a two speed economy, McKinsey Global Institute, marzo de 2014. 
Como puede verse, la precariedad (o informalidad laboral) distinguen a un grupo de los trabajadores mexicanos que se ubica en el sector inferior de la estructura del mercado laboral mexicano que concentra alrededor de $50 \%$ de la fuerza de trabajo activa en México. Este piso inferior se ubica principalmente (aunque no exclusivamente) en las entidades más pobres del país, entre los trabajadores más viejos y más jóvenes que apenas ingresaron al mercado laboral, en el sector rural y de servicios, y en los niveles de educación más bajos, pero también como vimos proporcionalmente más entre las mujeres que los hombres. Esta condición, además, está vinculada al tipo de unidad económica, de tal manera que la mayoría de los trabajadores de hasta doS SMD y con un trabajo informal o precario, laboran en micro y pequeños negocios.

\section{Distribución funcional del ingreso}

La caída del salario mínimo ha ido al parejo del conjunto de los salarios por lo menos hasta el comienzo del siglo xxI. Ello se ha reflejado también en la concentración del ingreso y en particular en el distribución funcional del ingreso. En el cuadro 14 se advierte esta tendencia desde 1982. Nótese que diez puntos porcentuales del PIB fue la pérdida que sufrieron los trabajadores en tan solo ocho años.

\begin{tabular}{|c|c|c|c|c|c|c|c|c|c|}
\hline \multicolumn{10}{|l|}{$\begin{array}{l}\text { Cuadro } 14 \\
\text { Salario real y }\end{array}$} \\
\hline & 1982 & 1983 & 1984 & 1985 & 1986 & 1987 & 1988 & 1989 & 1990 \\
\hline Salario real & 92.5 & 78.6 & 72.1 & 71.0 & 64.0 & 60.8 & 53.6 & 49.4 & 43.4 \\
\hline $\begin{array}{l}\text { Participación de los } \\
\text { salarios }\end{array}$ & 35.2 & 29.4 & 28.7 & 28.7 & 28.5 & 26.8 & 26.0 & 25.5 & 25.0 \\
\hline \multicolumn{10}{|c|}{$\begin{array}{l}\text { Fuente: INEGI, banco de Información económica, Cf. Moreno Brid y Ros, "Devolpment and } \\
\text { growth..." p. 154). }\end{array}$} \\
\hline
\end{tabular}

Por su parte, Márquez calcula que la desigualdad entre ambos factores tuvo una caída de ocho puntos entre 1982 y 2004 (cuadro 15).

\begin{tabular}{c|c|c|c|}
\multicolumn{2}{l}{ Cuadro 15 } \\
Ingreso nacional disponible = 100
\end{tabular}

Fuente: David Márquez, La Jornada, 8 de octubre de 2007. 
Los datos de INEGı más recientes nos muestran que la distribución factorial del ingreso ha permanecido casi constante entre 2005 y 2009 con una pequeña ventaja para los empleadores (cuadro 15).

\begin{tabular}{|c|c|c|c|c|c|}
\hline \multicolumn{6}{|c|}{$\begin{array}{l}\text { Cuadro } 15 \\
\text { Remuneración de los asalariados, sueldos y salarios, contribuciones de los empleadores y exce- } \\
\text { dente bruto de operación (en \%) 2005-2009 }\end{array}$} \\
\hline & 2005 & 2006 & 2007 & 2008 & 2009 \\
\hline Remun. Asalariados & 29.6 & 28.6 & 28.1 & 28.1 & 29.3 \\
\hline Sueldos y salarios & 26.6 & 25.7 & 25.2 & 25.2 & 26.3 \\
\hline Contribuciones sociales empleadores & 3.0 & 2.9 & 2.9 & 2.8 & 3.1 \\
\hline Impuestos & 10.3 & 10.3 & 10.1 & 12.9 & 9.6 \\
\hline Menos: subsidios & 0.4 & 0.8 & 0.8 & 2.3 & 0.4 \\
\hline Excedente bruto operación & 60.5 & 61.9 & 62.6 & 61.4 & 61.5 \\
\hline
\end{tabular}

Fuente INEGI Sistema de cuentas nacionales de México año base 2003 tomo

Este fenómeno no se presentó sólo en México. Según el informe sobre salarios de la оाт: “...desde el decenio de 1980, la mayoría de los países han experimentado una tendencia a la baja de la participación de los ingresos del trabajo, lo que significa que se ha destinado una proporción menor de la renta anual a la remuneración de la mano de obra y una proporción mayor a las rentas procedentes del capital". 14

La oIT advierte además que "si se excluye del cálculo la retribución de $1 \%$ superior de los trabajadores, la caída en la participación del trabajo resultaría mayor..."15

Según datos de un estudio de Stockhammer, 16 en los países desarrollados la parte del PIB correspondiente a los sueldos y salarios (cuota salarial) bajó entre 1980 y 2007 de 73.4 a 64\%. Por su parte, en los países en desarrollo la caída fue de 15 puntos porcentuales entre los años ochentas y los primeros años del siglo XXI, antes de la gran recesión de 2008.

Norma Samaniego 17 (2014) diseñó esta gráfica gracias a una investigación propia con datos de diversas instituciones que coincide con la caída de 15 puntos de Stockhammer pues, según la autora, la parte de los asalariados llegó en su punto más alto a $42.6 \%$ y luego bajó hasta 27.0 por ciento.

14 Organización Internacional del Trabajo (OIT), Informe mundial sobre salarios 2012/2013. Los salarios y el crecimiento equitativo Ginebra, 2013, p. 6.

15 oIT, op. cit. p. 47

16 Engelbert Stockhammer, "Why have wage shares fallen? A panel analysis of the determinants of functional income distribution Conditions of Work and Employment", Series No. 35, International Labour Office - Geneva, 2013, p. 3.

17 Samaniego, Norma "Desigualdad y mercado de trabajo en México". Conferencia, unAM, julio de 2014. 


\section{Gráfica 3}

La distribución funcional en México

Remuneraciones de asalariados/PIB

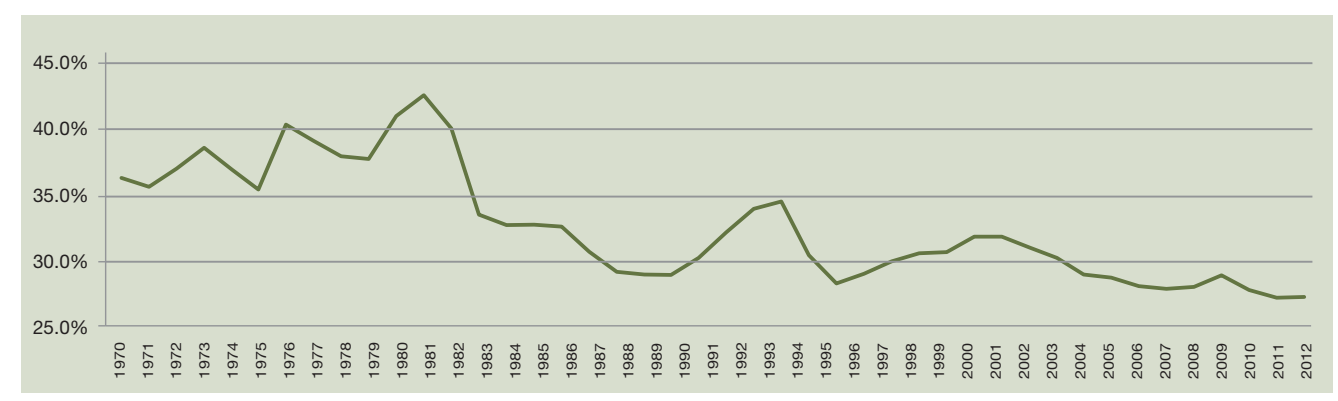

Fuente: Norma Samaniego. Elaboración propia con información histórica de INEGI, SCNM. Series encadenadas, 1979-1980, base 1979; 1980-1988, base 1980, 1988-2003, base 1993; 2003-2012, base 2008.

\title{
México un caso excepcional en América Latina (AL)
}

En la primera década del nuevo siglo, no todos los países de América Latina mantuvieron deprimidos los salarios mínimos legales. En este sentido, México ha sido una excepción. En el informe de la oIT sobre salarios 2013/2014, se destacan las diferencias entre México y Brasil en torno a la política salarial:

\begin{abstract}
"En América Latina, un país donde el salario mínimo ha tenido un impacto significativo es el Brasil. Aun cuando la estrategia de revaloración del salario mínimo se ha mantenido durante aproximadamente veinte años, se aceleró desde 2005 cuando, como parte de una estrategia para fomentar el consumo doméstico, los ajustes regulares se vincularon sistemáticamente a la inflación más el crecimiento del PIB. Esta misma estrategia se siguió aun durante los años de la crisis financiera, cuando la política salarial era parte de una estrategia anticíclica. Por el contrario, en México el salario mínimo aumentó solo modestamente en términos reales entre 2005 y 2011, debido a que la política de salario mínimo ha sido fuertemente determinada por los esfuerzos para lograr un equilibrio fiscal (ya que el salario mínimo determina numerosas prestaciones de seguridad social) y aumentar la competitividad exportadora. Como resultado, el salario mínimo está por debajo de los niveles del mercado, aun para los trabajadores no calificados. Estos dos casos ilustran los diferentes enfoques hacia el salario mínimo..."18
\end{abstract}

\section{En conclusión}

Si revisamos la evolución de los salarios mínimos en las últimas décadas encontramos dos etapas claramente definidas: 1954-1982 y 1982-2014. La primera de estas fases dura 28 años; la segunda lleva ya más de 30 años y aún no termina.

En estos períodos los salarios mínimos han sido un indicador de cómo se han movido el conjunto de la estructura salarial: entre 1950 y 1982, su aumento constante se inscribe en una economía en crecimiento, mientras que en el segundo periodo, su

18 oІт, "Informe Mundial..."2013, p. 42 
caída están relacionada con una economía que sufre crisis sucesivas de alto impacto y se mantiene con un crecimiento raquítico.

Así, en México, como en otras partes del mundo, los salarios crecieron (en la posguerra) como parte de un círculo virtuoso de expansión de la economía y luego tendieron a decrecer (a partir de los años ochenta). En esta segunda fase además ha aumentado el empleo informal (asalariado y por su cuenta) ${ }^{19}$ y la migración: todo ello ha provocado un empeoramiento general de las condiciones de trabajo. Está claro que en la primera fase hay un efecto que generaliza los beneficios, mientras que en la segunda hay un deterioro general de los ingresos de la mayoría de la población.

También hubo un cambio de paradigmas. Hasta los años ochenta se consideraba que un aumento de los salarios reales generaría un mayor crecimiento económico. Ahora se considera que el impulso económico proviene de la demanda externa. De esta manera, en la globalización neoliberal, debido al outsourcing o subcontratación,20 las políticas de ajuste y la velocidad de movimiento de los flujos de capitales, se dio una competencia por abaratar los salarios para mejorar la competitividad en el mercado mundial. El salario ya no se consideraba, como en el pasado, un factor de crecimiento sino una variable residual, que se mide por los índices de productividad. Ello propició además a flexibilizar el empleo y abaratar aún más el salario.

\section{Saldos de la política salarial}

El establecimiento de los salarios mínimos legales se originó en la Constitución de 1917, pero fue sólo hasta 1934 cuando se pusieron a funcionar las comisiones respectivas que entonces existían a nivel municipal. A partir de 1964, se creó la Comisión Nacional de los Salarios Mínimos (CONASAMI) que se apoyaba en una comisión regional para cada una de las 111 zonas económicas establecidas para la fijación de dichos salarios. A la fecha solo existen dos zonas económicas. ${ }^{21}$ Desde su creación y hasta ahora, la Comisión decreta los aumentos al salario mínimo legal y aunque se trata de un organismo de carácter tripartito, las decisiones del Ejecutivo se han impuesto en los hechos. No hay duda que la fijación de los salarios mínimos legales ha sido parte de una estrategia de política económica conducida por el gobierno en turno.

La experiencia de los últimos años nos muestra que mantener deprimido el salario mínimo ha ahondado las brechas salariales y la desigualdad. Ha propiciado igual-

19 Según las nuevas cifras del INEGI, la población ocupada informal alcanza a $60 \%$ del total, lo que representaba 13.7 millones de trabajadores en 2013, de los cuales 6.8 millones eran trabajadores asalariados. Se entiende que estos últimos no contaban con seguridad social ni con las prestaciones de ley.

20 Según datos de INEGI en el año 2008 el personal ocupado en las unidades económicas del sector privado y paraestatal que era "suministrado por otra razón social" alcanzaba la cifra total de 2.3 millones de trabajadores, mientras que el personal ocupado dependiente de la razón social sumaba 17.4 millones. Es decir, casi $12 \%$ del total estaban subcontratados. Llama la atención los casos de la industria manufacturera (13.6\% del total) y sobre todo la rama de servicios financieros y de seguros con $40.4 \%$ del total. Según diversas investigaciones, los trabajadores subcontratados gozan por lo general de bajos salarios y carecen de seguridad social y de las prestaciones de ley.

21 Cf. Banco de México, "Principales indicadores salariales", p. 10. 
mente que la proporción de ingresos que obtienen los trabajadores en relación a la que se apropian los empleadores haya disminuido.

La caída y luego el congelamiento del salario mínimo ha formado parte de una política salarial que ha provocado también una mayor pobreza laboral y que alienta la migración y la informalidad. La estructura de los salarios responde, por su parte, a una heterogeneidad estructural del aparato productivo pero la política laboral ha ampliado, no reducido, la precariedad laboral en su conjunto. Al mismo tiempo, se ha profundizado la debilidad institucional (en particular de las autoridades del trabajo) y la impunidad frente a las violaciones de la ley por parte de empresas formales e informales.

Cabe pensar entonces que un cambio en la política salarial podría inducir una nueva dinámica en la que un crecimiento de la demanda interna alentara la producción y por ende la productividad, y con ellos los niveles de empleo y de ingresos de la población. Esta otra dinámica se ha observado en diversos países de América Latina. $Y$ aunque las condiciones son naturalmente distintas que en el caso de México, puede afirmarse que en esos casos, el crecimiento del salario mínimo y (a una escala menor) de los salarios en su conjunto, fue parte de una estrategia diferente que propició un mayor bienestar a esas sociedades y disminuyó efectivamente la pobreza y la desigualdad.

Un aumento de los salarios mínimos en México tendría dos propósitos: en primer lugar, como dice la oIT, el objetivo sería tratar de reducir la pobreza y ofrecer mayor protección social a los trabajadores más vulnerables. Un salario mínimo deprimido afecta a quienes trabajan en peores condiciones, al nivel inferior del mercado laboral, a los más desprotegidos. Revertir esta situación ya sería un gran logro. Dejar las cosas como están sin duda haría aumentar el número de mexicanos que no obtienen los mínimos de bienestar por los ingresos que perciben a cambio de su trabajo.

En segundo lugar, aumentar el salario mínimo en términos reales, de manera gradual y moderada, podría influir positivamente en el nivel de vida de los trabajadores. Según diversos estudios, este incremento no debería afectar el empleo ni los niveles de precios. Por otra parte, su efecto sobre el conjunto de la estructura salarial y en la economía informal (el efecto faro) 22 de acuerdo a la experiencia internacional, sería reducido sobre todo en los salarios más elevados pero podría mejorar la distribución del ingreso y disminuir la desigualdad.

22 Cf. entre otros: John Schmitt, "Why does the minimum wage have no discernible effect on employment? " Center for Economic and Policy Research (CEPR), Washington, febrero de 2013; Raymundo Campos, "El salario mínimo y el empleo: Evidencia internacional y posibles impactos para el caso mexicano" , El Colegio de México, 2014. Sobre el efecto faro particularmente en la economía mexicana: David Kaplan y Francisco Pérez Arce Novaro "El efecto de los salarios mínimo en los ingresos laborales de México" El Trimestre Económico, vol. LxxIII, num. 289, enero-marzo de 2006. Sobre los efectos favorables de aumento del salario mínimo en los países en desarrollo, cf. Roxana Maurizio, "El impacto distributivo del salario mínimo en la Argentina, el Brasil,Chile y el Uruguay" CEPAL-Norwegian Ministry of Foreign Affairs, mayo de 2014; y Selim Pelek (Galatasaray University and París Nord University), "The impact of minimun wage on the wage distribution. Evidence from Turkey" (primary version), Journées de Microéconomie Appliquée, 2013. 


\section{Bibliografia}

Banco de México, Estadísticas Laborales, www.banxico.org.mx

Banco de México, Principales indicadores salariales, 2009.

Bolio, Eduardo y Jaana Temes, et al, A tale of two Mexicos: Growth and prosperity in a two speed economy, McKinsey Global Institute, marzo de 2014.

Bortz, Jeffry Lawrence, Los salarios industriales en la Ciudad de México 1939-1975. FCE, 1988.

CEPAL, Pactos para la lgualdad. Hacia un futuro sostenible, Trigésimo quinto período de sesiones de la CEPAL, Mayo de 2014.

Consejo Nacional de Evaluación de la Política Social coneval, Índice de Tendencia laboral de la Pobreza, 2014, www.conveal.gob.mx

Escobar Toledo, Saúl, Los Trabajadores en el Siglo xx. Sindicatos, Estado y Sociedad en México 19072004, UNAM, 2006

Garavito, Rosa Albina, Recuperar el salario real: un objetivo impostergable, ¿cómo lograrlo?. Friedrich Ebert Stiftung (FES), México, 2013.

Instituto Nacional de Estadística, Geografía e Informática (INEGI), El Colegio de México. Estadísticas

Económicas del Porfiriato. Fuerza de Trabajo y Actividad Económica por Sector.

INEGI, Indicadores Estratégicos de Ocupación y Empleo (www.inegi.org.mx)

INEGI, Encuesta Nacional de Ocupación y Empleo.

INEGI, Sistema de cuentas nacionales Tomo I.

Márquez David, “La Distribución del Ingreso y el gasto en México”, La Jornada, 8 de octubre de 2007.

Moreno Brid, Juan Carlos y Jaime Ros, Development and Growth in the Mexican Economy. A historical perspective, Oxford University Press, 2009

Organización Internacional del Trabajo (OIT), "Informe mundial sobre salarios 2012/2013. Los salarios y el crecimiento equitativo" Ginebra, 2013.

Reyes Heroles G.G., Jesús, La distribución de los Ingresos por Trabajo en México, Banco de México, 1981. Samaniego, Norma, "Desigualdad y mercado de trabajo en México". Conferencia dictada en la unam, Grupo

Nuevo Curso de Desarrollo, julio de 2014.

Secretaría de Trabajo y Previsión Social, Estadísticas del sector www.stps.gob.mx

Stockhammer, Engelbert, "Why have wage shares fallen? A panel analysis of the determinants of functional income distribution Conditions of Work and Employment", Series núm. 35, International Labour Office -Geneva, 2013

Tello, Carlos, Sobre la desigualdad en México. unam, Facultad de Economía, México, 2010. 